\title{
Is There a Role for Magnetic Resonance Imaging in Diagnosing Palpable Breast Masses When Mammogram and Ultrasound Are Negative?
}

\author{
Molly L. Olsen, M.D., Marilyn J. Morton, D.O., ${ }^{2}$ Daniela L. Stan, M.D., ${ }^{1}$ and Sandhya Pruthi, M.D.
}

\begin{abstract}
Background: The use of breast magnetic resonance imaging (MRI) for screening high-risk patients is well established. However, the role of MRI as a diagnostic problem-solving tool is less well studied. With the increasing availability of MRI, its use for problem solving has increased. This small retrospective study examines the use and utility of breast MRI in evaluating palpable breast masses with negative diagnostic mammogram and ultrasound studies.

Methods: We reviewed our breast MRI database, selecting breast MRI studies performed to assess palpable abnormalities with negative mammogram and ultrasound findings. Evidence of cancer was determined by biopsy.

Results: Seventy-seven studies were included, comprising 1.3\% of all breast MRI studies performed at our institution during the study period (2005-2011). Twenty-two patients underwent biopsy, and 55 were followed clinically without biopsy. Approximately half (27 of 55) of the patients without biopsy were lost to follow-up after negative MRI, and the rest had no evidence of cancer on imaging or clinical examination at 1 year. Of the 22 patients who underwent biopsy, 2 were diagnosed with cancer, both with positive MRI studies. Sensitivity of MRI when compared to tissue diagnosis was $100 \%$, and specificity was $70 \%$. Positive and negative predictive values were $25 \%$ and $100 \%$, respectively.

Conclusions: When used for evaluation of a palpable breast mass with negative traditional imaging, breast MRI likely offers low yield of cancer diagnosis and low specificity. Negative MRI results may cause a low compliance rate for recommended follow-up. Because a biopsy is indicated for persistent palpable masses, the addition of diagnostic MRI only adds another step, with associated costs and burdens.
\end{abstract}

\section{Introduction}

B REAST CANCER IS A LEADING ONCOLOGIC cause of death in women, and early detection saves lives. Breast magnetic resonance imaging (MRI) has been shown to be highly sensitive in the detection of breast cancer but only moderately specific because of an overlap in enhancement features of benign and malignant breast lesions. ${ }^{1}$ Thus, this costly examination results in false positive findings that generate additional diagnostic examinations and biopsies, increasing the cost of care for the patient. ${ }^{2}$ As responsible use of healthcare dollars becomes increasingly important, appropriate use of breast MRI is under debate. The role of MRI in screening women at high risk for breast cancer is well defined, ${ }^{1}$ but the use of breast MRI as a problem-solving tool is less well defined. $^{2-5}$
Breast MRI as a problem-solving tool has been studied recently in the setting of suspicious, yet inconclusive, traditional (mammography and ultrasound) imaging studies ${ }^{2,3}$ and when worrisome clinical signs and symptoms exist. ${ }^{2}$ These studies demonstrate that MRI adds little to the diagnostic workup when mammogram or ultrasound shows concerning findings, and it remains unclear how MRI might be useful in the setting of negative conventional imaging results and persistent suspicious clinical concerns. Most treatment algorithms for the workup of a breast mass advocate biopsy or reassurance with short-term follow-up if conventional imaging is negative. ${ }^{6,7}$ The choice of follow-up vs. biopsy is generally dictated by the level of clinical suspicion. Multiple treatment guidelines widely available in popular media advocate the use of MRI in helping make that decision, ${ }^{8,9}$ and MRI is used on occasion for this purpose in practice, although

${ }^{1}$ Department of Medicine, Division of General Internal Medicine, and ${ }^{2}$ Department of Radiology, Mayo Clinic, Rochester, Minnesota. 
to our knowledge, there are no data to support its use in this context. $^{2}$ Furthermore, the question of whether breast MRI may have higher accuracy in the setting of various clinical symptoms, such as palpable masses, breast pain, or nipple discharge, remains unanswered. To help guide appropriate use of healthcare spending, this study aims to determine the diagnostic utility of breast MRI in detecting cancer in palpable breast masses with negative conventional imaging.

\section{Materials and Methods}

\section{Patient selection}

The protocol for this retrospective chart review was approved by the Mayo Clinic Institutional Review Board. We queried our prospectively populated MRI database for all breast MRI studies performed at our institution from January 1, 2005, through April 21, 2011, for the clinical indication "persistent breast clinical concern with negative imaging." The listed indications for MRI ordering were determined at the time of testing by the clinical physician for the purpose of patient care, without research intention, and recorded prospectively. It is possible that the indication was selected by the ordering physician during the episode of care for a variety of problems, yet only a few were pertinent to this study.

We selected only the cases with the clinical indication that fit criteria from the available database. We included breast MRI examinations performed to evaluate palpable breast abnormalities when diagnostic mammogram and ultrasound studies performed within 90 days earlier were negative. Mammogram and ultrasound reports were considered negative if the original report's assessment was benign or negative (Breast Imaging-Reports and Data System [BI-RADS] 1 or 2). If ultrasound or mammogram findings were inconclusive or suspicious enough to prompt the radiologist to recommend MRI, those cases were excluded. The MRI studies were interpreted as positive or negative based on the original radiologist's interpretation. Whether MRI findings corresponded to the anatomic location of the palpable abnormality or represented incidental findings in other areas of either breast was recorded.

MRI studies performed in high-risk patients (based on family or personal history of breast cancer or $B R C A$ gene mutation) were included only if the MRI was obtained for diagnostic assessment of the palpable mass rather than for screening purposes. We included studies of patients who had other breast concerns (e.g., breast erythema, pain, or nipple discharge) only if a palpable mass was noted to be the primary reason for obtaining the MRI studies. We excluded MRI studies performed for the following indications: high-risk screening, staging of previously biopsy-proven cancer, nipple discharge or retraction without palpable mass, and palpable breast mass evaluation when a mammogram or ultrasound was not obtained within 90 days or was not interpreted as negative. Studies were also excluded if the patient had previously documented refusal of access to records for research purposes.

\section{Data collection}

Medical charts were reviewed by four physicians involved in our multidisciplinary breast practice. Demographic data, pathology and radiology reports, and clinical notes were reviewed in the electronic medical record. The locations of palpable abnormalities and MRI findings were recorded to determine concordance. Cases posing uncertainty were reviewed by a second reviewer and by a third if the first two opinions differed. De-identified data were collected and stored in a secure and private database.

Biopsy-proven pathologic tissue examination provided definitive diagnosis of a malignant or benign finding. Infiltrating or invasive carcinoma and ductal carcinoma in situ were considered malignant. Atypical hyperplasia was recorded when present and was considered benign for this study. All other biopsy findings were considered benign. Biopsy-compared data were used to calculate MRI sensitivity and specificity and positive and negative predictive values for breast cancer detection. Confidence intervals (CI) of 95\% were calculated. Breast biopsy was considered the gold standard, and only cases where a breast biopsy of the concerning lesion was performed were included in the calculations. Biopsies were performed within 60 days of the breast MRI in all cases but 1 . In this case, the patient underwent mastectomy for risk reduction 7 months after the breast MRI, and all breast tissue was analyzed. Patients who did not undergo biopsy were considered to be without evidence of cancer if conventional imaging was negative and clinical notes indicated no interval diagnosis or signs or symptoms of breast cancer at least 1 year after MRI. These patients were not included in sensitivity and specificity calculations, as no biopsy results were available for gold standard comparison. Follow-up adherence rates were recorded from the medical chart review. Monetary charges to patient accounts were estimated with figures provided by our institution's business office.

\section{Breast MRI technique}

All breast MRI examinations were performed in a 1.5-Tesla magnet (General Electric Medical Systems, Milwaukee, WI) using a dedicated breast coil (USA Instruments, Aurora, $\mathrm{OH}$ ) with the patient in the prone position. Scan acquisitions were performed before and after administration of a single dose of gadolinium-based contrast agent with a calculated dose of 0.1 $\mathrm{mmol} / \mathrm{kg}$ body weight. Since 2005, power injection has been used with a rate of $3 \mathrm{~mL} / \mathrm{sec}$, followed by a saline flush.

Dynamic imaging sequences included one precontrast and five postcontrast T1-weighted fat-suppressed 3D spoiled gradient-recalled echo series. The first postcontrast acquisition was acquired within 2 minutes after contrast administration, followed by five sequential delayed acquisitions every 60-90 seconds. Since 2007, scans have been acquired in the axial plane with a repetition time / echo time (TR/TE) of 6.9/ 2.8 , flip angle of $10^{\circ}$, field of view (FOV) 24-38, slice thickness of $1.8 \mathrm{~mm}$, and matrix size to keep the in-plane resolution to $<1.0 \mathrm{~mm}$. Prior to 2007, scans were acquired in the sagittal plane. For image interpretation, precontrast and postcontrast subtraction images were used in addition to source image analysis in all studies. Since 2006, examinations have been processed and interpreted with the use of a computer-aided detection system (CADstream, Confirma, Kirkland, WA).

\section{Results}

Figure 1 outlines the flow of patients through the study. A total of 5769 breast MRI examinations were performed at our 


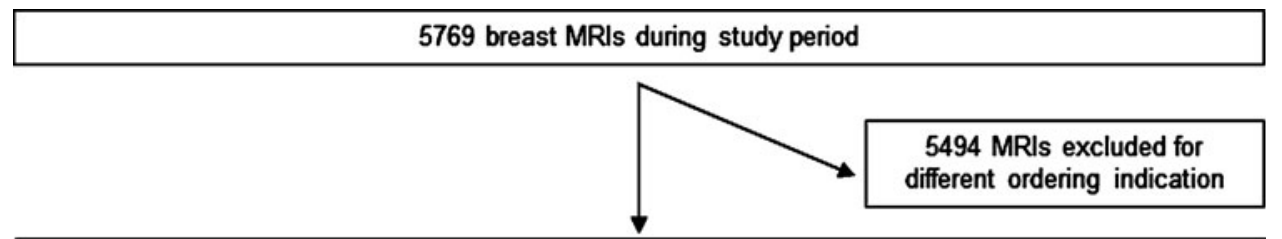

276 breast MRI studies performed for persistent clinic breast concern with negative imaging

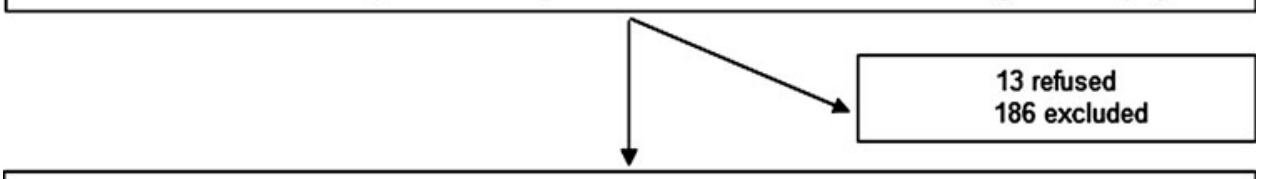

77 cases of palpable breast concern with negative mammogram and ultrasound within 90 days

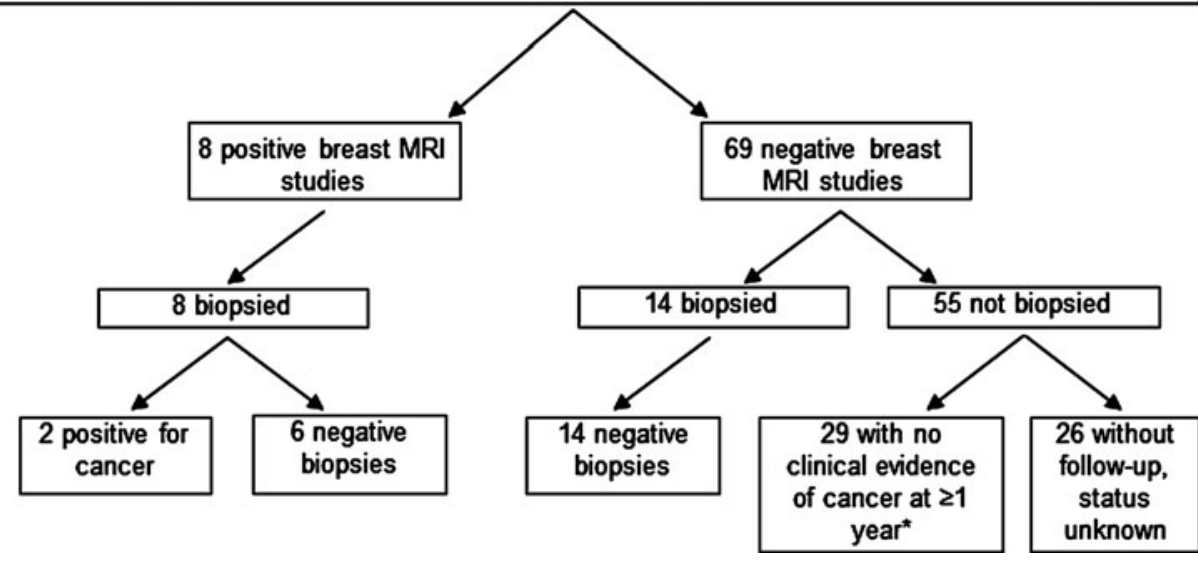

institution during the study period. Of those examinations, $276(5 \%)$ were performed for the indication, "persistent clinical breast concern with negative imaging." Thirteen of those were excluded because of patient refusal of consent for research use of medical records. Of the remaining 263 cases, 186 were excluded because of lack of negative conventional imaging within 90 days or ordering indication not fitting the inclusion criteria. Thus, 77 cases were included, comprising roughly $1.3 \%$ of all breast MRI studies performed at our institution during the study period.

Of the 77 patients whose breast MRI studies were included, all patients were women, and the average age was 52, range 31-84. Forty-eight percent were at high risk for developing cancer, half with a strong family history of breast cancer and the other half with a personal history of breast cancer. No patients carried known BRCA gene mutations. Of the included 77 MRI studies, 8 were positive for suspicious imaging findings corresponding to the anatomic location of the palpable abnormality, and all 8 underwent biopsy. One biopsy was performed by excision, and the rest were performed with a core needle (3 without imaging assistance, 4 with MRI guidance). Of the 8 biopsies of palpable abnormalities, 2 cancers were revealed. Sensitivity of MRI for detecting cancer was $100 \%$ (CI 16\%-100\%). No other cancers in different anatomic areas from the palpable masses were discovered incidentally on initial MRI.

Patient 1 was 61 years old, with a history of invasive ductal carcinoma of the left breast treated with lumpectomy and whole breast irradiation 2 years earlier. She complained to her oncology provider of left subareolar breast mass. Breast ultrasound and diagnostic mammogram showed benign
FIG. 1. Flow of patients through study. There was no biopsy confirmation of a benign breast condition in this group. MRI, magnetic resonance imaging.

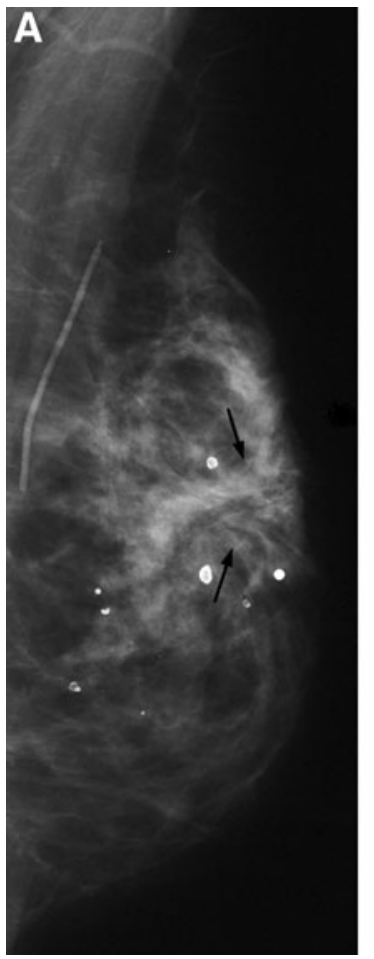

B

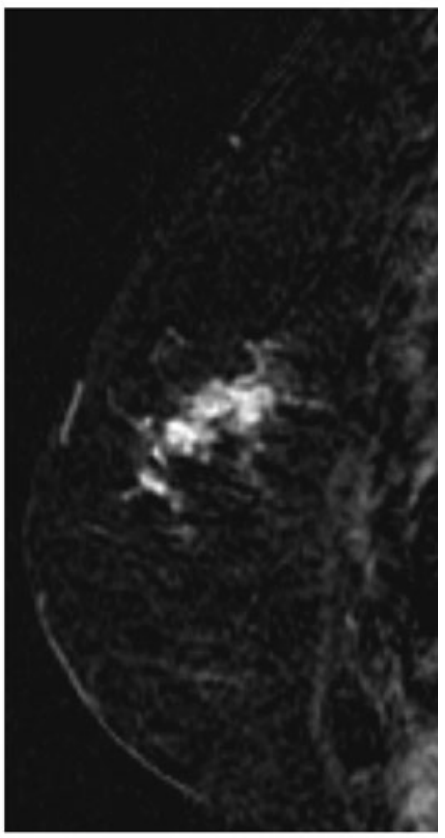

FIG. 2. (A) Left medial-lateral-oblique view (MLO) mammogram shows dense breast tissue and postoperative distortion (arrows) at the lumpectomy site in the upper left breast. Targeted ultrasound of the palpable lump in this area was negative. (B) Left sagittal postcontrast subtraction MR image shows segmental nonmass enhancement in the upper breast corresponding to the palpable lump. Palpationdirected needle biopsy revealed invasive ductal carcinoma. 

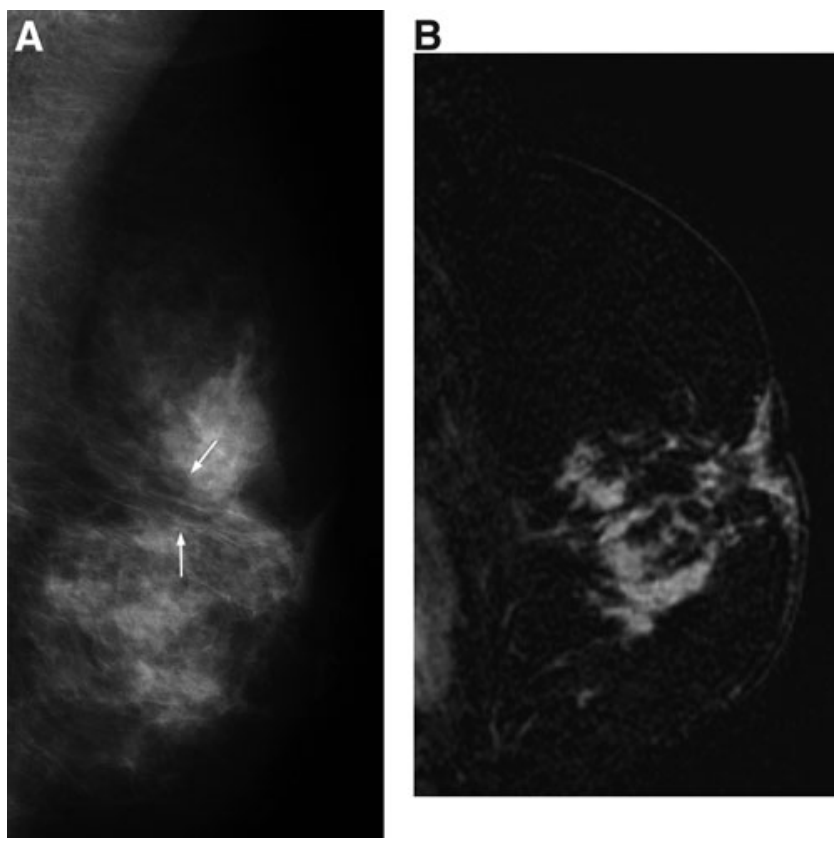

FIG. 3. (A) Left MLO mammogram shows dense breast tissue and postoperative distortion (arrows) at the lumpectomy site in the upper central left breast. Initial targeted ultrasound of the palpable lump in this area was negative. (B) Left sagittal postcontrast subtraction MR image shows segmental nonmass enhancement in the central breast corresponding to the area of the palpable lump. A second-look ultrasound showed an ill-defined hypoechoic area, and needle biopsy revealed invasive mixed ductal and lobular carcinoma.

findings, including postoperative architectural distortion, appearing stable compared with previous examinations (Fig. 2A). The mass remained persistently bothersome to the patient, and she sought further evaluation. Breast MRI was performed 62 days later, revealing a 5-cm area of abnormal enhancement in the left breast corresponding to the palpable area. Core needle biopsy revealed grade II/III adenocarcinoma with mixed ductal and lobular features (Fig. 2B). After a modified radical left mastectomy, pathology review confirmed a 7-cm malignancy with cellular types noted above and with 8 of 8 axillary lymph nodes bearing metastatic disease. Patient 1 had traditional imaging initially performed at another institution, and the hard copy films are no longer available.

Patient 2 was a 59-year-old woman with a history of left breast invasive ductal carcinoma treated with lumpectomy and whole breast irradiation 5 years earlier. She came to the breast clinic complaining of a new lump near her surgical scar. Diagnostic mammogram and ultrasound showed no suspicious findings (Fig. 3A). The mass remained bothersome to the patient and physician. MRI was performed 20 days later and revealed a $4-\mathrm{cm}$ area of enhancement corresponding to the palpable area of concern in the left breast. Core needle biopsy revealed invasive ductal carcinoma (Fig. 3B). The patient underwent left modified radical mastectomy, and final pathology showed the malignancy spanned $4 \mathrm{~cm}$, with two internal mammary lymph nodes containing metastatic disease. The remaining 6 patients with positive MRI studies had benign findings on biopsy; thus, the positive predictive value of MRI was $25 \%$ (CI $3 \%-65 \%$ ). One of the patients with a false positive MRI result had atypical ductal hyperplasia detected on MRI-guided core needle biopsy. No cancer was present in the lesion after surgical excision.

The other 69 MRI studies were negative. Of the women with negative MRI studies, 14 underwent biopsy (10 by MRIguided core needle procedure, 4 by excision) despite the negative MRI study, and pathology studies revealed benign tissue. Specificity was 70\% (CI 46\%-88\%). The negative predictive value was 100\% (CI 77\%-100\%). The other 55 women with negative breast MRI studies were recommended to follow-up later, with imaging and clinical evaluation. In 26 (47\%) of those cases, the outcome could not be assessed because the patients did not return to our institution as recommended. In the remaining 29 women who did return for follow-up, no evidence of cancer was found later, although no biopsies were performed. Incidental findings on MRI led to 6 additional follow-up MRI studies, as well as 21 additional second-look and follow-up ultrasound studies. The net patient charges for initial and subsequent studies for all included patients totaled roughly $\$ 386,000$.

\section{Discussion}

To our knowledge, this small study is the first to examine the frequency of breast MRI use and evaluate its diagnostic utility for cancer diagnosis in palpable breast masses with negative conventional imaging. In this study, we found that this clinical indication accounted for $1.3 \%$ of all breast MRI studies performed at our institution during the study period. Only a small percentage of patients who had breast MRI performed underwent diagnostic biopsy. When compared to biopsy, MRI had high sensitivity $(100 \%)$ but lower specificity $(70 \%)$ and low positive predictive value (25\%) for diagnosing breast cancer. Because of the small sample size, the CIs were wide, indicating low precision. When breast MRI was negative, our patients showed a disconcertingly low compliance rate for recommended follow-up evaluation. Our findings of high sensitivity and low specificity of breast MRI for diagnosing palpable abnormalities are consistent with those of another recent study that assessed MRI problem-solving utility in patients with various breast symptoms and abnormal, rather than reassuring, traditional breast imaging. ${ }^{2}$ It is certainly possible that a larger sample size would find a lower sensitivity and even lower specificity.

In this study, overall diagnostic yield of cancer was low. This finding could in part be due to bias introduced by the small sample size of our study but is consistent with other studies. ${ }^{2}$ It is likely that these results reflect a low prevalence of breast cancer in this specific population because combined mammogram and ultrasound have been shown to have a high negative predictive value. ${ }^{10}$ Furthermore, given the high cancer risk and persistently concerning nature of the masses of the 2 women diagnosed with cancer, MRI could have been avoided and biopsy of the palpable lesion performed directly.

Because of the irregular texture of breast tissue, patients often seek medical attention for palpable breast findings, and clinicians must decide whether examination findings warrant further evaluation. Persistent palpable masses of clinical concern, even despite negative 
conventional imaging, should undergo biopsy. Patients who have physical examination findings that are thought to represent benign fibroglandular breast tissue can be offered reassurance and short-term follow-up if traditional imaging is negative.

Presumably, MRI was requested for patients in our study to provide additional guidance in determining if the clinical suspicion warranted additional evaluation, likely in cases posing uncertainty. It is also possible that patient anxiety in this study was a driving force in pursuing MRI of some breast masses rather than the true clinical concern of the clinical breast examination. This could not be quantified in this study with available resources. At our institution, MRI is widely available, but in most practices, this may not be the case. Most of our MRI studies were negative, not surprisingly, given the high negative predictive value of combined mammogram and ultrasound. ${ }^{10} \mathrm{~A}$ minority of the patients underwent biopsy despite a negative breast MRI. In these cases, a negative MRI study failed to provide enough reassurance to prevent a biopsy, thus adding no clear benefit to the patients' workup but instead adding other burdens as well as the cost of the test itself. Furthermore, MRI led to no downstream cancer diagnosis of incidental findings in this series. If the palpable findings were persistently concerning enough to prompt consideration of further advanced imaging, no modality of imaging would have provided reassurance enough to forego the gold standard biopsy. As a biopsy is indicated for persistent palpable masses, ${ }^{7}$ the addition of diagnostic MRI only adds another step with associated costs and burdens.

The majority of patients with palpable abnormalities and negative imaging were recommended for clinical follow-up and did not undergo tissue diagnosis. We found an alarmingly high rate of noncompliance for recommended followup after negative MRI studies, which likely results from the reassurance some patients and physicians may have gained from the negative MRI findings. Unfortunately, we do not know if any of those patients may were found later to have breast cancer. In part, the poor follow-up compliance was also likely due to the fact that a large portion of our patients live far from our facility, secondary to referral patterns of our practice. Thus, some patients may have chosen to establish care with local providers. In addition, patients included in this study were from other states, making it challenging to obtain long-term data on subsequently diagnosed breast cancers.

This study was limited by retrospective design, small sample size, limited follow-up, and single institution participation. Furthermore, by the nature of the chart review study design, we were unable to capture the clinical outcome of many patients. Larger-scale reviews with comparison to national tumor registries would ensure accurate follow-up results. We do not advocate prospective data collection using MRI and mandatory biopsy in masses with negative conventional imaging to further answer this question because of the high cost and morbidity of such a study. However, larger-scale, multisite retrospective studies would help further determine if our findings are consistent with those of other practices. Comparisons with women with traditional imaging and palpable breast masses who did not undergo breast MRI would also be informative.
The overall use of breast MRI for further evaluation of palpable masses at our institution was not insignificant and caused substantial cost. We believe breast MRI could have been avoided in all the cases reviewed. We have used these data for internal quality improvement measures to prevent unnecessary MRI imaging, and we hope they may be used similarly in other practices.

In summary, we found that MRI had relatively high sensitivity and low specificity for detecting cancer in suspicious palpable breast masses with negative conventional imaging. However, because of the small sample size, precision was low. The overall cancer yield was low and absenteeism for recommended return visits was high after negative MRI findings. The use of breast MRI for this indication comprised a small, but not insignificant, portion of all breast MRI indications, and breast MRI use resulted in significant costs and burdens.

\section{Conclusions}

We do not advocate using breast MRI instead of biopsy for the diagnostic workup of concerning palpable masses with negative conventional imaging, as the likelihood of diagnosing cancer is low, the test is costly, and negative MRI results may provide false reassurance leading to missed or delayed diagnosis through avoiding biopsy. We advocate that if MRI is being considered in diagnosing a breast mass, clinical suspicion is likely high enough to warrant biopsy of the palpable mass instead. Further investigation is needed to determine appropriate use of MRI in the evaluation of other clinical breast problems, including nipple discharge and breast pain, as MRI may be more useful in settings in which the possible biopsy location is unknown given the lack of palpable findings.

\section{Acknowledgments}

We thank the Mayo Clinic Department of Medicine and Division of General Internal Medicine for supporting this study and the Center for Translational Science Activities for study design and statistical support.

\section{Disclosure Statement}

No competing financial interests exist.

\section{References}

1. Saslow D, Boetes C, Burke W, et al. American Cancer Society guidelines for breast screening with MRI as an adjunct to mammography. CA Cancer J Clin 2007;57: 75-89.

2. Yau EJ, Gutierrez RL, DeMartini WB, Eby PR, Peacock S, Lehman CD. The utility of breast MRI as a problem-solving tool. Breast J 2011;17:273-280.

3. Moy L, Elias K, Patel V, et al. Is breast MRI helpful in the evaluation of inconclusive mammographic findings? Am J Roentgenol 2009;193:986-993.

4. Keller DL. Radiologic workup of a palpable breast mass. Cleve Clin J Med 2009;76:385.

5. Orel S. Who should have breast magnetic resonance imaging evaluation? J Clin Oncol 2008;26:703-711. 
6. Stein L, Chellman-Jeffers M. The radiologic workup of a palpable breast mass. Cleve Clin J Med 2009;76:175-180.

7. Pruthi S. Detection and evaluation of a palpable breast mass. Mayo Clin Proc 2001;76:641-647.

8. Mayo Clinic staff. Why it's done, 2011. Mayo Foundation for Medical Education and Research. Available at www .mayoclinic.com/health/breast-mri/MY00300/DSECTION = why-its-done Accessed November 2, 2011.

9. A physician's guide. Clinical indications for breast MRI. Southern Tier Imaging. Available at www.stmri.com/pdf/ STI_Indication\%20Breast\%20CI.pdf Accessed November 2, 2011.
10. Soo MS, Rosen EL, Baker JA, Vo TT, Boyd BA. Negative predictive value of sonography with mammography in patients with palpable breast lesions. Am J Roentgenol 2001;177:1167-1170.

Address correspondence to: Sandhya Pruthi, M.D. Mayo Clinic 200 First Street SW Rochester, MN 55905

E-mail: pruthi.sandhya@mayo.edu 